\title{
DIE VERWENDUNG VON SPIELEN FÜR DEN UNTERRICHT VON ENGLISCH ALS ZWEITSPRACHE
}

\section{REVIEW ARTICLE}

DIAS, Adailton Di Lauro ${ }^{1}$

DIAS, Adailton Di Lauro. Die Verwendung von Spielen für den Unterricht von Englisch als Zweitsprache. Revista Científica Multidisciplinar Núcleo do Conhecimento. Jahrgang 04, Ed. 07, Vol. 03, S. 69-76. Juli 2019. ISSN:2448-0959

\section{ABSTRAKT}

Die Studierenden, die die aktuellen Lehrpraktiken bewerten und sogar historisch methodische Aspekte beobachten, haben heute große Schwierigkeiten, die kognitiven Fähigkeiten zu entwickeln und Wissen im aktuellen Bildungssystem zu erwerben. Vielleicht liegt das nicht an der Unwirksamkeit der Methoden, sondern an der Dynamik der Beziehungen zwischen Student und Umwelt. Verschiedene Altersgruppen erfordern unterschiedliche Ansätze, die sich in den Zustand der Obsoleszenz geändert haben; sie verlieren ihre Wirksamkeit deutlich, wie die Zeit vergeht. So können die Spiele in Bezug auf Alter und Themen eine effiziente Möglichkeit sein, Inhalte aufgrund ihrer Universalität anzuzeigen. Der vorliegende Artikel zielt darauf ab, die Gründe zu erklären, warum die Spiele ein wichtiges Instrument in der Bildung darstellen, und einige Möglichkeiten aufzuzeigen, wie diese Werkzeuge im Lehr- und Lernprozess von Englisch als Zweitsprache angewendet werden können.

Schlüsselwörter: Spiel, Fähigkeit, Sprache, Emotionen, Lernprozess.

\footnotetext{
${ }^{1}$ Master es Student in Science Education an der Unigrendal University. Fachmann für Englisch von FIJ-Integrated Colleges of Jacarepagua, Rio de Janeiro (2007). Literaturschwerpunkt an der University of State of Bahia, Brasilien. Englisch und Portugiesisch Lehrer bei IFRR-Brasilien.
} 


\section{EINFÜHRUNG}

Angesichts des kontinuierlichen technologischen Fortschritts und noch mehr unter Beachtung, dass die Methoden diese Veränderungen mit dem Ziel einer ständigen Verbesserung begleiten sollten, versucht dieser Artikel, die Verwendung von Spielen mit der Praxis des Englischunterrichts als Zweitsprache. Die Studie beschränkt sich nicht auf ein bestimmtes Modell des Spiels, sondern auf jede spielerische Art, die Realität zu simulieren. So beschreibt die Analyse einige Arten von Spielen und ihre historischen Aspekte, die die enge Beziehung zwischen innen, dem Kontext und dem Lernprozess feststellen. Der derzeitige Vorschlag kann zu einer Schaffung von Praktiken konvergieren, die Spiele nutzen, um den Lernakt für die Schüler angenehm zu machen; es kann innen auch helfen, die vorgeschlagenen Inhalte fließend und effizient in ihrem täglichen Leben zu nutzen.

Der Mangel an realen Situationen, die zur Praxis der neuen Sprache durch die Schüler beitragen, stellt eine der größten Schwierigkeiten für den Englisch-Unterricht dar. Im Rahmen des Klassenzimmers können verschiedene Aktivitäten einen Teil dieser Lücke schließen, wenn der Lehrer das Interesse der Schüler am Lernen entwickelt und sich auf die expositiven Klassen und Aktivitäten konzentriert. Betrachtet man die vielen Möglichkeiten in einer Umgebung, in der die Schüler außerschulische Situationen simulieren und in den Kontext dieser Situationen eintreten können, kann ein besseres Ergebnis erzielt werden. Die Perspektiven für die konzeptionelle Ordnung in dieser Studie berücksichtigen die angegebene Literatur, aber vor allem berücksichtigt die Erfahrung des Autors in der pädagogischen Praxis, vor allem im Unterrichten von Englisch als Zweitsprache. Daher ersetzen die Sicht und Beobachtung des Autors in dieser Studie die übliche erforderliche Feldforschung.

Basierend auf Forschungen von Spielen für Kinder, Spielen und anderen Konzepten und verwandten Themen, gibt es verschiedene Arten der Anwendung dieser Studie im pädagogischen Bereich. Für Dantas (1998) "bezieht sich der Begriff lächerlich auf den Akt des Spielens (auf freie und individuelle Weise) und des Spiels (in Bezug auf ein soziales Verhalten, das Regeln beinhaltet)." Die spezifische und dynamische Beziehung zwischen der Methode und den Schülern macht jedoch die Vertiefung und 
die Richtung der Spiele für jedes Fach notwendig. Nach ANTUNES (2003), um die Spiele für die Praxis des effektiven Lehrens anwendbar zu machen, ist es notwendig, Analyse und Forschung, da das Ergebnis umgekehrt werden kann, wenn es keine Kompatibilität zwischen innen und dem Ziel des Lernens. Die verwendete Literatur führt einen Teil der Konzeptkonstruktion, ohne die Anwendbarkeit der Spiele zu definieren. Der Autor ist also derjenige, der eine solche praktische Anwendung und seine mögliche methodische Konvergenz vorschlägt.

\section{EINIGE ÜBERLEGUNGEN ZU DEN LERNSCHRITTEN}

Ein wichtiger Punkt, der berücksichtigt werden muss, ist der Kontext und die Bedingungen, die auf die Lernmechanismen angewendet werden. In Bezug auf die Spiele ist das Publikum sehr heterogen, angesichts der Vielfalt der bestehenden Spiele und unbegrenzte Reichweite im Zusammenhang mit dem Alter der Schüler. Das Spiel ist eine spontane Aktivität, die von einem oder mehreren Menschen durchgeführt wird und, anders als Kunst und Arbeit, kann als mittels körperlicher oder geistiger Stimulation betrachtet werden, manchmal beides. Die Existenz der Spiele geht auf prähistorische Zeiten zurück und deckt alle Erfahrungsstufen, Geschlechter und Altersgruppen ab. Unter den Lernstadien ist es wichtig zu verstehen, dass Überlebenshindernisse die Rechtfertigung für die verschiedenen Prozesse sind. Daher treten diese Prozesse in einem Umfang zunehmender Komplexität auf. Die Schaffung von Operationen, die notwendig sind, um diese Hindernisse zu überwinden, durchläuft verschiedene und klar definierte Phasen, von der Kindheit bis zur Jugend.

Die erste Stufe beginnt bei der Geburt und dauert in der Regel bis zum Beginn des Spracherwerbs (bis etwa 18 Monate alt). Während dieser Zeit beginnen die Bildung von motorischen Fähigkeiten und Sinneswahrnehmung. Die zweite Stufe tritt etwa zwischen zwei und vier Jahren alt auf und wird durch die Bildung symbolischen Denkens definiert. Die Verwendung von Puppen, Autos und anderen Objekten symbolischer Natur, die die Realität simulieren, sind Teil dieser besonderen Stufe. Zwischen vier und acht Jahren erscheint die dritte Stufe, in der sich der intuitive Gedanke zu bilden beginnt und die Objekte um sie herum zu Bezugspunkten werden. 
Automatisch führt diese Intuition zur vierten Stufe, der Phase, in der Menschen lernen, konkrete Operationen zu organisieren, um jedes Objekt, das auf seine Bedeutung verweist, zu assoziieren. Die vierte Phase dauert zwischen vier und acht Jahren. Die letzte Phase ist in der Pubertät abgeschlossen, wo der Aufbau von mentalen Operationen für analytisches Denken ausreicht. Mit diesen Schritten und ihren Unterscheidungen ist es möglich zu verstehen, dass die Angemessenheit von Spielen für verschiedene Altersgruppen die meisten ihrer Mitglieder treffen kann.

\section{VERWENDUNG DER GAMES ALS EINE UNTERSTÜTZUNG FÜR TEACHING ENGLISH}

Für WITTGENSTEIN (2001) kann sich der Begriff "Spiel" nicht auf eine einzige Definition beschränken, sondern als eine Vielzahl von Einstellungen, um ein Verhältnis der Familiären Ähnlichkeit zwischen innen herzustellen. Die Definition der Vielfältigkeit von Wittgenstein findet auch in CALLOIS (1957) Unterstützung, die einige notwendige Merkmale für eine Aktivität als Spiel definiert. Die Bedingungen sind, dass die Aktivität Spaß machen muss, zeitlich und ortsbegrenzt, unvorhersehbare Ergebnisse, und das ist nicht produktiv. Darüber hinaus muss die Tätigkeit unterschiedliche Regeln des täglichen Lebens haben und fiktiv sein, begleitet vom Bewusstsein einer anderen Realität. Die Spiele müssen sich daher dem Realen an das Imaginäre und an das Klassenzimmer nähern, oder der gewählte alternative Raum muss ein geeignetes Umfeld für diese Praktiken sein.

Die Verwendung von Wiederholung in den Sprachniveaus, beim Englischunterricht, sollte als Unterrichtsprozess der Muttersprache erfolgen, auch für Anfänger. Abgesehen vom Grammatiklernen ist die Technik des Englischlernens mit dem Zeigefinger, der auf Bilder oder Objekte zeigt, sehr effizient. Der Lehrer muss die Präsentation der Sprache klar und in Spielen durchführen, sollten Sie Aktivitäten vermeiden, die zu Langeweile oder Ablenkung führen. Aufgaben wie Übersetzung und Grammatik haben zunächst kaum einen wirklichen Wert, da die geschriebene Sprache an dieser Stelle zweitrangig ist. Das Hauptziel ist es, die Sprache mit dem Kontext und der realen, abstrakten oder fiktiven Umgebung von Spielen zu verknüpfen. 
Die Auswahl der Spiele sollte sich an der Anwendbarkeit auf die betreffende Altersgruppe, der technischen Durchführbarkeit und dem Stand der Überwachung durch den Lehrer in allen Phasen der Tätigkeit orientieren. So können Bau- oder Lehrpädagogen-Lernspiele verwendet werden, da sie die oben genannten Anforderungen erfüllen. Je nach Zweck kann jede Art von Spiel je nach verschiedenen Bedingungen verwendet werden, aber es ist wichtig, das Interesse der gesamten beteiligten Gemeinschaft zu erfassen. Im Folgenden sind einige Arten von definierten Spiel, die verwendet werden können:

\subsection{INTERAKTIVE UND WECHSELNDE SPIELE}

Diese Spiele werden verwendet, um dem Mitglied einer Gruppe beizutreten. Sie helfen den Teilnehmern, sich spezifische Informationen zu merken und eine entspannte Atmosphäre zu fördern. Die Spieler werden abgelenkt, entwurmt, lösen Spannungen und überwanden persönliche Reserven. Sie müssen in den ersten Phasen der Entwicklung einer Gruppe verwendet werden, wenn ein Treffen beginnt, nach einer Pause und jedes Mal, wenn die Gruppe zu beginnen müde, gelangweilt oder nicht motiviert.

Musik und Tanz werden in aktiven Spielen empfohlen, um die Gruppe auf die vorgeschlagene Aktivität einzustimmen. Die ideale Entscheidung ist, kurze Spiele mit viel Action und einem hohen Energieaufwand zu verwenden.

\subsection{SPIELE VON TOUCH UND VERTRAUEN}

Diese Spiele helfen den Teilnehmern, sich selbst mit dem Vertrauen in ihr Leben auseinanderzusetzen. Nach der Kulturorganisation und dem Grad der Öffnung der Menschen, kann die Gruppe nach und nach zu anderen Übungen gehen, die Berührung beinhalten. Die Spiele der Berührung und des Vertrauens müssen mit viel Sorgfalt verwendet werden; der Instruktor muss auf den Moment und die Reaktionen der Gruppe sowie der Teilnehmer achten und innen Ressourcen anbieten, um gut mit starken internen psychologischen Prozessen umzugehen. 


\subsection{SPIELE DER KREATIVITÄT UND REFLEXION}

Dies sind Spiele, die imaginären Ausdruck, Intuition und Kreativität stimulieren. In diesen Spielen können sich die Teilnehmer selbst bemerken und anderen offen zeigen, was sie über sich selbst, über das studiumende Thema und über die Gruppe entdeckt haben. Die Spieler kommen mit ihrem Inneren und dem Inneren anderer Spieler in Kontakt und bemerken, was in allen Levels relevanter ist. Diese Art von Spiel muss verwendet werden, wenn die Gruppen vollständig integriert sind, zusammenarbeiten und alle Bedingungen nutzen, um bis zu diesem Zeitpunkt tief in das untersuchte Thema einzutauchen.

\subsection{SPIELE DES MANAGEMENTS}

Diese Spiele konzentrieren die Aufmerksamkeit der Spieler auf die Planung, Verwaltung von Ressourcen, Simulation von Situationen und das Erlernen spezifischer Techniken. Diese Art von Spiel muss verwendet werden, nachdem die Gruppe gut integriert ist, damit sie zum vorgeschlagenen Ziel gelangen kann. Manchmal ist es üblich, einige Schwierigkeiten beim Erlernen des Themas zu erscheinen, so ist es sehr wichtig, dass der gesamte lebende Lernzyklus vollständig gearbeitet wird.

\subsection{ABSCHLUSSSPIELE}

Diese Spiele helfen Menschen, ihre Position in Bezug auf ein Thema, auf die Gruppe und auf sich selbst zu stellen und das, was sie während des Lernprozesses zu haben, in ihren Alltag zu übertragen. Die Synthesizer- und Endspiele sind diejenigen, die ritualisieren, bewerten und formalisieren, was während der Arbeit getan wurde. Es ist wirklich wichtig, jedem Teilnehmer klarzumachen, dass ein Zyklus beendet wird und ein anderer beginnt.

\subsection{GESCHICKLICHKEITS- UND STRATEGIESPIELE}

Kann in jedem Schritt des Prozesses oder Situationen verwendet werden. Es ist wichtig zu betonen, dass der Sport in fast allen oben erläuterten Modalitäten enthalten 
ist, aber er muss verwendet werden, um die Gruppe über den Aspekt des Lernens zu beteiligen, ohne jedoch den Wettbewerbsaspekt aufzugeben, der genau das ist, was die Öffentlichkeit anzieht. die meisten Altersgruppen. Das Spiel, als eine Aktivität zum Vergnügen gehalten, stellt sich als ein Werkzeug zu studieren und angewendet werden, um Englisch-Unterricht vor allem, weil es bekannt ist, dass unser Gehirn lernen durch einen Prozess der Wiederholung, Versuch und Geschwindigkeit (VILLA \& SANTANDER, 2003). Der Versuch oder das Experimentieren ist die Analyse der Situation. Die Wiederholung gibt die Praxis, die zu der Geschwindigkeit führt, oder Effizienz und Wirksamkeit. Die Fähigkeit jedes Schülers wird zeigen, welches Spielniveau verwendet werden sollte.

Wenn man also die unterschiedlichen Definitionen von Spielen und die Frage nach den Grundsätzen des Lernens vom Menschen versteht, wird die Bedingung der vollen Nutzung in den Lehrpraktiken deutlich. Das Interesse der Schüler am Lernen wird mit seinem Interesse an dem vorgeschlagenen Spiel zu dieser Zeit verbunden. Die durch das Spiel geschaffene Umgebung, die auch berücksichtigt, dass die Emotionen, die den Menschen beeinflussen, auch die Assimilation fördern, kann den Mangel an realen Situationen liefern. Je höher die Aufregung in den Spielen ist, desto größer ist die Möglichkeit, den vorgeschlagenen Inhalt festzulegen.

Positive Emotionen fördern mehr Lernen (SISTO \& MARTINELLI, 2006), so dass das Gefühl der Freude die Traurigkeit oder den Schmerz überwindet. Der Lehrer beginnt in der Zwischenzeit als Vermittler zwischen dem Realen und der Phantasie zu fungieren, die durch das Spiel und sein Ergebnis repräsentiert wird. Das Klassenzimmer verschiebt sich von einem Ort des pädagogischen Diskurses zu einem Labor für den Erfahrungsaustausch, voller Möglichkeiten.

\section{SCHLUSSFOLGERUNG}

Diese Studie versuchte, eine Alternative zum englisch-pädagogischen Prozess als Zweitsprache durch Spiele zu bieten, um Werte zum aktuellen Bildungskontext zu aggregieren. Das Lehren einer Sprache aus ihrer natürlichen Umgebung ist ein Prozess, der Von den beteiligten Fachkräften Geschicklichkeit und Kreativität 
erfordert. Dann, vom Moment der Auswahl der Spiele bis zur Analyse der Ergebnisse, sollte der Lehrer darauf achten, die Entwicklungen der Anerkennung der Schüler zu überwachen, um das eigentliche Ziel nicht zu verlieren. Der Fokus liegt nicht nur auf der Entwicklung von Strategien, sondern auch darauf, dass sie richtig funktionieren.

Sicherlich ist die Sache noch nicht zu Ende. Das ständige Studium und der Erfahrungsaustausch liegen weiterhin in der Verantwortung der am Lehr- und Lernprozess beteiligten Personen. Die mehr oder wenigere Nutzung und Verbreitung des Verfahrens, in dem die vorgeschlagenen Praktiken angewendet werden, wird den Erfolg dieser Verfahren bestimmen. Den Bildungsraum als etwas zu verbessern und kann entsprechend seiner Dynamik die statischen und veralteten Konzepte nicht halten.

\section{VERWEISE}

ABERASTURY, A. A criança e seus jogos. Porto Alegre: Artes Médicas, 1992.

AFFONSO, R. M. Ludodiagnóstico. São Paulo: Plêiade, 1995.

AGUIAR, J. S. Jogos para o ensino de conceitos: leitura e escrita na pré-escola. Campinas: Papirus, 1998.

ANTUNES, C. Jogos para a estimulação das múltiplas inteligências. $12^{a}$ edição. Petrópolis, RJ: Vozes, 2003.

CALLOIS, Roger. Les Jeux et les hommes. Paris: Gallimard, 1957.

DANTAS, H. Brincar e Trabalhar. In: KISHIMOTO, T. M. (org). Brincar e suas teorias. São Paulo: Pioneira, 1998.

SISTO, F. F. \& MARTINELLI, S. de C. (orgs.) Afetividade e dificuldades de aprendizagem: uma abordagem psicoeducacional. São Paulo: Vetor, 2006.

VILA, Magda \& SANTANDER, Marli. Jogos cooperativos no processo de aprendizagem acelerada. São Paulo: Qualitymark, 2003 
WITTGENSTEIN, Philosophical Investigations, Blackwell Publishing Ltd., MA: 2001.

Eingereicht: Juni 2019..

Aprovate: Juli 2019. 